CASE NOTES

\title{
MICROCORNEA AND CONGENITAL COLOBOMA OF IRIS AND CHOROID IN ONE EYE WITH CONGENITAL GLAUCOMA AND CORNEAL OPACITY IN THE OTHER EYE*
}

\author{
BY \\ S. P. SRIVASTAVA \\ G. R. Medical College, Gwalior, India
}

Case Report

A female baby 12 days old was brought to the out-patients department of Hamidia Hospital, Bhopal (M.P.), the parents complaining that the child was born with a white opacity in the right eye. She was the third child, the other two being alive and healthy with no congenital defects. The mother gave no history of any intercurrent illness during pregnancy. The child was full-term and the delivery was normal. There was no family history of any congenital ocular anomaly and the parents' eyes were normal.

Examination.-The right eye was enlarged as a whole. The cornea was large and slightly protruding, of a horizontal oval shape, more enlarged in the horizontal than in the vertical meridian. There was a dense diffuse opacity involving the whole of the cornea. Only on the lateral side, where the opacity was less dense, could the iris be faintly seen, almost in contact with the posterior surface of the cornea, as if the anterior chamber were absent. No other details of the anterior chamber, iris, pupil, or lens could be seen because of the dense corneal opacity. The eyeball was hard to the touch and the ocular tension was $60 \mathrm{~mm}$. $\mathrm{Hg}$ (Schiötz).

The left eye was slightly microphthalmic, with microcornea, and a typical iris coloboma. The cornea was clear. A lenticular opacity was seen in the upper part of the coloboma, but through the lower clear portion a coloboma of the retina and choroid was visible in the typical position. No other congenital anomaly was present.

The general appearance of the two eyes is shown in the Figure.

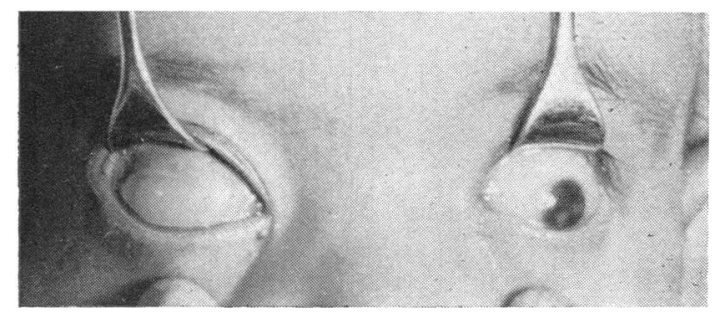

FIGURE-Opaque cornea of right eye and microphthalmos of left eye.

* Received for publication December 13, 1960. 


\section{Comment}

Microcornea, with congenital coloboma of the iris and choroid, and congenital glaucoma are not uncommon congenital abnormalities. The former may occur in association with a number of other congenital defects in one or both eyes, but its association with congenital glaucoma and corneal opacity in the other eye of the same individual is quite unusual.

The congenital glaucoma in the right eye in this patient seems to be due to a gross defect in the development of the anterior chamber and its angle, which has caused a type of buphthalmos with a very shallow or absent anterior chamber and marked corneal opacification. It is difficult to think of any aetiological relationship between the two conditions in the two eyes.

\section{Summary}

A case of microcornea, with congenital coloboma of the iris, retina, and choroid in the left eye, and congenital glaucoma and corneal opacity in the right eye has been described. It is very unusual to find two such abnormalities in the same patient. 